\title{
Minimally invasive papillary muscle sling placement during mitral valve repair in patients with functional mitral regurgitation
}

\author{
Orlando Santana, MD, ${ }^{\mathrm{a}}$ Natalia V. Solenkova, MD, ${ }^{\mathrm{b}}$ Andres M. Pineda, MD, ${ }^{\mathrm{a}}$ Christos G. Mihos, DO, ${ }^{\mathrm{a}}$ and \\ Joseph Lamelas, $\mathrm{MD}^{\mathrm{c}}$
}

\begin{abstract}
Background: We evaluated the safety and feasibility of minimally invasive mitral valve repair with papillary muscle sling placement via a right anterior thoracotomy approach in patients with severe functional mitral regurgitation (MR).
\end{abstract}

\begin{abstract}
Methods: We retrospectively reviewed all minimally invasive mitral valve repairs with papillary muscle sling placement in patients with severe functional MR performed at our institution between October 2011 and September 2012. The operative times, lengths of stay, postoperative complications, and mortality were analyzed.

Results: We identified a total of 19 consecutive patients. There were 12 men (63\%); the mean age was $60 \pm 13$ years. The mean \pm SD left ventricular ejection fraction was $23 \% \pm 5.5 \%$, and $4(21 \%)$ of the patients underwent previous coronary artery bypass graft surgery. The median aortic cross-clamp and cardiopulmonary bypass times were 106 (interquartile range [IQR], 76-120) and 163 (IQR, 119-170) minutes, respectively. The median intensive care unit length of stay was 64 (IQR, 43-75) hours, and the median postoperative length of stay was 7 (IQR, 5-7.5) days. Postoperatively, 2 patients developed acute kidney injury. There were no reoperations for bleeding or any cerebrovascular accidents. The 30-day mortality was 0 . A follow-up echocardiogram, obtained at a median of 3 (IQR, 1-7.5) months, demonstrated none to trivial MR in all patients.
\end{abstract}

Conclusions: Minimally invasive mitral repair with papillary muscle sling placement for severe functional MR is safe and effective in the short-term. Long-term data are needed to evaluate the effects on left ventricular remodeling and to assess the durability of the repair. (J Thorac Cardiovasc Surg 2014;147:496-9)

In patients with left ventricular systolic dysfunction and functional mitral regurgitation (MR), the surgical treatment of the mitral insufficiency is a challenging issue. Most of these individuals have a structurally normal mitral valve, but the valve is incompetent because left ventricular remodeling has disturbed the relationship between the subvalvular apparatus and the mitral valve leaflets. Therefore, more attention is given to correcting the papillary muscle displacement when these individuals undergo mitral valve repair. One of these approaches involves the placement of a sling around the papillary muscles. This technique has been previously reported as being performed via median sternotomy. We report our experience performing this technique using a minimally invasive approach via a right lateral thoracotomy.

\section{METHODS}

After obtaining approval from the Mount Sinai Medical Center (Miami Beach, Fla) Institutional Review Board, with patient consent waived, we

From the Columbia University Divisions of Cardiology, ${ }^{\mathrm{a}}$ Internal Medicine, ${ }^{\mathrm{b}}$ and Cardiac Surgery, ${ }^{\mathrm{c}}$ Mount Sinai Heart Institute, Miami Beach, Fla.

Disclosures: Authors have nothing to disclose with regard to commercial support.

Received for publication Jan 7, 2013; revisions received Feb 12, 2013; accepted for publication March 5, 2013; available ahead of print April 1, 2013.

Address for reprints: Orlando Santana, MD, Echocardiography Laboratory, Columbia

University Division of Cardiology, Mount Sinai Heart Institute, 4300 Alton Rd,

Miami Beach, FL 33140 (E-mail: osantana@msmc.com).

0022-5223/\$36.00

Copyright (c) 2014 by The American Association for Thoracic Surgery

http://dx.doi.org/10.1016/j.jtcvs.2013.03.006 retrospectively reviewed all heart operations performed at our institution between November 2011 and September 2012, to identify patients with functional MR who underwent mitral valve repair with papillary muscle sling placement using a minimally invasive approach.

All patients had their valvular lesions documented by diagnostic catheterization and echocardiography, and all operative reports and echocardiograms were reviewed. Intraoperative transesophageal echocardiography was performed to evaluate the mitral valve, and grading of the MR was done in accordance with the American Society of Echocardiography guidelines. ${ }^{1}$ The MR was graded as severe $(4+)$, moderate to severe $(3+)$, moderate $(2+)$, mild $(1+)$, or trace/none $(0)$. A postoperative transesophageal echocardiogram was obtained to evaluate the repair. The surgical technique time was analyzed on the basis of aortic cross-clamp and total cardiopulmonary bypass times. The outcome variables evaluated were 30-day mortality and postoperative complications, which were identified as follows: postoperative renal failure, bleeding requiring reoperation, cerebrovascular accident, prolonged ventilation ( $>24$ hours), reintubation, deep wound infection, and atrial fibrillation. The definitions and variables selected were based on the Society of Thoracic Surgeons Database definitions. All patients were evaluated approximately 30 days postoperatively in the outpatient setting.

\section{Surgical Technique}

A femoral platform was used to establish cardiopulmonary bypass. A longitudinal 2- to $3-\mathrm{cm}$ incision was made superior to the inguinal crease. The femoral artery was cannulated with a $16 \mathrm{~F}$ to $18 \mathrm{~F}$ arterial cannula (Edwards, Irvine, Calif) or a $15 \mathrm{~F}$ to $19 \mathrm{~F}$ arterial cannula (Bio-medicus; Medtronic, Minneapolis, Minn), and the femoral vein was cannulated with a $25 \mathrm{~F}$ venous cannula (Bio-medicus, Medtronic). With the aid of transesophageal echocardiography, the venous cannula was placed in the superior vena cava. A 5- to 6-cm skin incision was made in the right fourth to fifth intercostal space lateral to the anterior axillary line. A soft tissue retractor and rib spreader were used to provide further exposure. The 


\section{Abbreviations and Acronyms \\ $\mathrm{IQR}=$ interquartile range \\ $\mathrm{MR}=$ mitral regurgitation}

pericardium was opened over the phrenic nerve and tacked to the skin. By using transesophageal echocardiographic guidance, a retrograde coronary sinus catheter was inserted into the right atrium directly through the incision. One dose of antegrade cold blood cardioplegia was given to establish electromechanical arrest of the heart. Thereafter, retrograde cold blood cardioplegia was given throughout the procedure at 20-minute intervals. A left lateral atriotomy is performed through the Waterson groove to enter the left atrium. An atrial lift retractor and atrial exposure blade were used for visualization of the mitral valve. A specially designed papillary exposure device was used to visualize the infravalvular apparatus. We do not place the mitral annular sutures first because this impairs visualization. A longshafted curved clamp was used to encircle all sets of papillary muscles. This procedure needs to be performed carefully to not penetrate the papillary muscles. In addition, the clamp needs to be as close to the base of the papillary muscles as possible. Different size clamps are required depending on the width of the muscles. This maneuver can be time-consuming for it to be performed properly. This will avoid the graft slipping superiorly and entrapping the chordae. A 4-mm polytetrafluoroethylene graft (Gore-Tex; W. L. Gore \& Associates, Inc, Newark, Del) was then placed around the base of the papillary muscles (Figure 1). Care is taken to place the graft so that it has an anchoring muscle that will prevent upward migration. The edges of the sling are approximated as tightly as possible and tied with a 4-0 Prolene suture (Ethicon, Inc, Somerville, NJ) in a mattress manner and then a continuous over-and-over manner. This may require several sutures to apply the maximum tension to approximate the graft. At the completion, the papillary muscles are tightly approximated and juxtaposed. Thereafter, the mitral valve repair was performed. The size of the anterior leaflet was used to determine the size of the annuloplasty ring. We do not undersize the ring. The annuloplasty ring used was a profile threedimensional ring (Medtronic). A 4-0 Prolene suture was used to close the left atrium. Carbon dioxide was infused into the operative field at $2 \mathrm{~L} / \mathrm{min}$ throughout the entire procedure.

When concomitant tricuspid valve surgery was performed, the superior and inferior vena cavae were encircled with vessel loops. Before the right atrium was opened, the long femoral venous cannula was withdrawn into the inferior vena cava and then both cavae were snared with the vessel loops. The right atrium was then opened, and a sump suction was inserted into the superior vena cava to provide drainage. We did not use a separate venous cannula.

In patients with a history of coronary artery bypass surgery, moderate to deep hypothermia $\left(24^{\circ} \mathrm{C}-26^{\circ} \mathrm{C}\right)$ and fibrillatory arrest were used. Cardioplegia was not delivered at all. Removal of air was performed via a vent placed through the atriotomy into the left ventricle and an aortic root vent.

In patients with a history of atrial arrhythmias, a concomitant modified left atrial ablation procedure was performed first after exposure of the left atrium and mitral valve. The intra-atrial ablation lines were created with a saline irrigated unipolar radiofrequency probe (Cardioblate; Medtronic). The ablation lines included isolation of the pulmonary veins, followed by a box lesion communicating the pulmonary veins. Additional lines were created around the left atrial appendage, and from the appendage to the left pulmonary veins. The last lesion set was from the left pulmonary veins to the mitral annulus (P2-P3 region). The left atrial appendage was ligated from within the left atrium with a double row, continuous suture line. We did not perform right atrial ablation in any of the patients.

With the heart empty, a ventricular pacing wire was placed. After discontinuing cardiopulmonary bypass and administering protamine, decannulation was performed. The purse string sutures were tied, and the femoral artery was reinforced using a 5-0 Prolene suture. A single chest tube was left in the pleural space. For pain relief, an On-Q pain relief system was inserted (I-Flow Corporation, Lake Forest, Calif). Two catheters were placed to continuously deliver $0.25 \%$ bupivacaine for 72 hours. The thoracotomy incision was closed in the routine manner.

\section{Statistical Methods}

Continuous variables are expressed as the mean $\pm \mathrm{SD}$ or median and interquartile range (IQR, 25th-75th quartile), as appropriate. The statistical analyses were performed using SPSS, version 17 (SPSS Inc, Chicago, Ill).

\section{RESULTS}

We identified 19 consecutive patients, consisting of 12 $(63 \%)$ men and $7(37 \%)$ women, with a mean age of $60 \pm 13$ years. The mean ejection fraction was $23 \% \pm 5.5 \%$, with a mean mitral valve tenting height of $10 \pm 2 \mathrm{~mm}$. There were $4(21 \%)$ patients with a history of coronary artery bypass graft surgery. In all patients, the MR was functional, with an ischemic cause in $9(47 \%)$ and a nonischemic origin in $10(53 \%)$. All patients were in New York Heart Association Functional class III to IV (Table 1). Of the 19 patients, 2 underwent concomitant tricuspid valve repair due to the presence of moderate to severe tricuspid regurgitation and 3 underwent a concomitant ablation procedure for atrial fibrillation.

The median aortic cross-clamp and cardiopulmonary bypass times were 106 (IQR, 76-120) and 163 (IQR, 119-170) minutes, respectively. On postoperative transesophageal echocardiogram, none of the patients had any discernible MR. The median number of units of packed red blood cells transfused was 1 (IQR, 0-2). The median intensive care unit length of stay was 64 (IQR, 43-75) hours. Postoperatively, 2 $(10.5 \%)$ patients developed renal failure. None of the patients had a cerebrovascular accident, required reoperation for bleeding, or developed a deep wound infection. The median postoperative length of stay was 7 (IQR, 5-7.5) days (Table 2). There were no operative deaths, and at a median follow-up of 9.5 (IQR, 6.5-12) months, all patients were alive.

A follow-up echocardiogram, obtained at a median of 3 (IQR, 1-7.5) months, demonstrated none to trivial MR in all patients. The mean ejection fraction increased to $31 \% \pm 17 \%$, and the mitral valve tenting height was reduced to a mean of $5 \pm 2 \mathrm{~mm}$.

\section{DISCUSSION}

Functional, or secondary, mitral insufficiency in patients with reduced left ventricular systolic function is usually a result of annular dilatation and papillary muscle displacement, with the mitral leaflets being anatomically normal. ${ }^{2}$ Whether to perform mitral valve surgery in patients with severe MR and advanced heart failure is controversial, with the published data showing equivocal results. ${ }^{3-5}$ Thus, to our knowledge, the optimal approach to the management of individual patients with functional MR has not been 


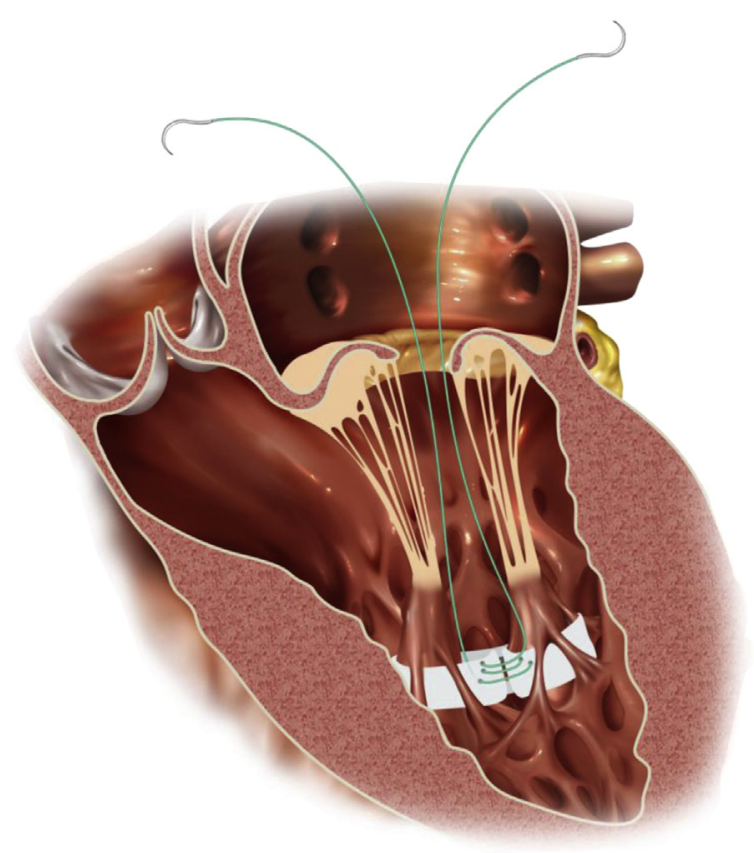

FIGURE 1. Placement of a 4-mm polytetrafluoroethylene (Gore-Tex) tube around the papillary muscles.

established. Presently, the most common type of surgery performed in these cases is an undersized mitral annuloplasty, which was popularized by Bolling and colleagues. ${ }^{6}$ In this technique, a mitral annuloplasty ring that is downsized by 2 sizes is used to cause a reduction in the anteroposterior (septolateral) diameter of the mitral valve, increasing the surface of coaptation. The drawback with this technique is that it has a high rate of failure, with reports of up to $28 \%$ of patients developing moderate or more MR within 6 months postrepair. ${ }^{7}$

To reduce the recurrence of MR in these patients, some authors have focused their attention on fixing the

TABLE 1. Patient baseline characteristics

\begin{tabular}{lc}
\hline \multicolumn{1}{c}{ Variables } & Value $(\mathbf{N}=\mathbf{1 9})$ \\
\hline Age, $y^{*}$ & $60 \pm 13$ \\
Male sex & $12(63)$ \\
Hypertension & $18(95)$ \\
Diabetes mellitus & $6(32)$ \\
Dyslipidemia & $14(74)$ \\
Ejection fraction, \%* & $23 \pm 5.5$ \\
Previous coronary bypass graft surgery & $4(21)$ \\
History of cerebrovascular accident & $2(10.5)$ \\
History of atrial fibrillation & $7(37)$ \\
Chronic obstructive pulmonary disease & $4(21)$ \\
Preoperative creatinine level, mg/dL* & $1.0 \pm 0.3$ \\
Ischemic cardiomyopathy & $9(47)$ \\
Nonischemic cardiomyopathy & $10(53)$ \\
New York Heart Association functional class III-IV & $19(100)$ \\
\hline
\end{tabular}

Values are given as number (percentage) unless otherwise indicated. *Values are given as mean $\pm \mathrm{SD}$.
TABLE 2. Operative results and postoperative outcomes

\begin{tabular}{lc}
\multicolumn{1}{c}{ Variables } & Value $(\mathbf{N}=\mathbf{1 9})$ \\
\hline Aortic cross-clamp time, min & $106(76-120)$ \\
Cardiopulmonary bypass time, min & $163(119-170)$ \\
PRBCs transfused, U & $1(0-2)$ \\
Intra-aortic balloon pump use* & $2(11)$ \\
Total ventilator time, h & $16(10.5-22)$ \\
Intensive care unit length of stay, h & $64(43-75)$ \\
Renal failure* & $2(10.5)$ \\
Prolonged intubation* & $4(21)$ \\
Atrial fibrillation* & $1(5)$ \\
Hospital length of stay, d & $7(5-7.5)$ \\
\hline Values are given as median (interquartile range) unless otherwise indicated. No \\
patients experienced reoperations for bleeding, cerebrovascular accidents, reintuba- \\
tions, sternal deep wound infections, or 30-day mortality. PRBC, Packed red blood \\
cell. *Values are given as number (percentage).
\end{tabular}

subvalvular apparatus in conjunction with the standard annuloplasty ring repair. Hvass and colleagues ${ }^{8,9}$ were the first to report the use of a 4-mm polytetrafluoroethylene tube encircling the trabecular base of both papillary muscles in patients with ischemic functional MR. A moderately undersized mitral annuloplasty ring was initially placed in most patients, with the last 10 patients of the study receiving a normally sized prosthetic mitral ring. All patients also underwent coronary artery bypass graft surgery. At 1 year follow-up, there was an improvement in ejection fraction, a reduction in the end-diastolic and end-systolic diameters, and a reduction in the mean pulmonary artery systolic pressure.

All of the previously mentioned procedures were done via a standard median sternotomy. Minimally invasive valve surgery, when compared with median sternotomy, has been shown to reduce surgical trauma and decrease morbidity, especially in high-risk patients, such as elderly and obese persons. ${ }^{10-14}$ Therefore, it is tempting to hypothesize that, by placing a papillary muscle sling during mitral valve repair via a minimally invasive approach, one may improve the short- and long-term results of the mitral valve repairs in these high-risk individuals. Another benefit of using a minithoracotomy approach is that visualization of the infravalvular apparatus is improved. At our institution, the patients we select for this procedure are those with severe functional MR who have an ejection fraction of $40 \%$ or less. Although the median aortic cross-clamp time was 106 (IQR, 76-120) minutes, this did not seem to have an adverse effect on outcomes.

\section{Study Limitations}

The current study is subject to the limitations inherent in a single-center, retrospective study design. The sample size is small, and the median follow-up echocardiographic period is limited, being only 3 months. Therefore, longerterm data are needed to be able to compare the recurrence 
of MR of this approach with the recurrence that has been noted when undersized mitral annuloplasty is used. Furthermore, all operations were performed by a single surgeon (J.L.). Thus, our findings can only be cautiously generalized. Finally, this study does not address the clinical indications for mitral surgery in patients with severe left ventricular dysfunction and functional mitral insufficiency.

\section{CONCLUSIONS}

The placement of a papillary muscle sling during minimally invasive mitral valve repair via a right anterior thoracotomy for severe functional MR is safe and effective in the short-term. Long-term data are needed to evaluate the outcomes of these patients and to assess the durability of the mitral valve repair.

\section{References}

1. Zoghbi WA, Enriquez-Sarano M, Foster E, Grayburn PA, Kraft CD, Levine RA, et al. Recommendations for evaluation of the severity of native valvular regurgitation with two-dimensional and Doppler echocardiography. J Am Soc Echocardiogr. 2003;16:777-802.

2. Levine RA, Schwammenthal E. Ischemic mitral regurgitation on the threshold of a solution: from paradoxes to unifying concepts. Circulation. 2005;112:745-58.

3. Wu AH, Aaronson KD, Bolling SF, Pagani FD, Welch K, Koelling TM. Impact of mitral valve annuloplasty on mortality risk in patients with mitral regurgitation and left ventricular systolic dysfunction. J Am Coll Cardiol. 2005;45:381-7.
4. Bishay ES, McCarthy PM, Cosgrove DM, Hoercher KJ, Smedira NG, Mukherjee D, et al. Mitral valve surgery in patients with severe left ventricular dysfunction. Eur J Cardiothorac Surg. 2000;17:213-21.

5. Gummert JF, Rahmel A, Bucerius J, Onnasch J, Doll N, Walther T, et al. Mitral valve repair in patients with end stage cardiomyopathy: who benefits? Eur J Cardiothorac Surg. 2003;23:1017-23.

6. Bolling SF, Pagani FD, Deeb GM, Bach DS. Intermediate-term outcome of mitral reconstruction in cardiomyopathy. J Thorac Cardiovasc Surg. 1998;115: 381-8.

7. McGee EC, Gillinov AM, Blackstone EH, Rajeswaran J, Cohen G, Najam F, et al. Recurrent mitral regurgitation after annuloplasty for functional ischemic mitral regurgitation. J Thorac Cardiovasc Surg. 2004;128:916-24.

8. Hvass U, Tapia M, Baron F, Pouzet B, Shafy A. Papillary muscle sling: a new functional approach to mitral repair in patients with ischemic left ventricular dysfunction and functional mitral regurgitation. Ann Thorac Surg. 2003;75:809-11.

9. Hvass U, Joudinaud T. The papillary muscle sling for ischemic mitral regurgitation. J Thorac Cardiovasc Surg. 2010;139:418-23.

10. Lamelas J, Sarria A, Santana O, Pineda AM, Lamas GA. Outcomes of minimally invasive valve surgery versus median sternotomy in patients age 75 years or greater. Ann Thorac Surg. 2011;91:79-84.

11. Santana O, Reyna J, Grana R, Buendia M, Lamas GA, Lamelas J. Outcomes of minimally invasive valve surgery versus standard sternotomy in obese patients undergoing isolated valve surgery. Ann Thorac Surg. 2011;91:406-10.

12. Cohn LH, Adams DH, Couper GS, Bichell DP, Rosborough DM, Sears SP, et al Minimally invasive cardiac valve surgery improves patient satisfaction while reducing costs of cardiac valve replacement and repair. Ann Surg. 1997;226 421-6.

13. Gammie JS, Zhao Y, Peterson ED, O'Brien SM, Rankin S, Griffith BP. Less-in vasive mitral valve operations: trends and outcomes from the society of thoracic surgeons adult cardiac surgery database. Ann Thorac Surg. 2010;90:1401-10.

14. Gammie JS, Bartlett ST, Griffith BP. Small-incision mitral valve repair: safe, durable, and approaching perfection. Ann Surg. 2009;250:409-15. 